\title{
Analytical nonlocal model for shear localization in wall- bounded dense granular flow
}

\author{
Keng-Lin Lee ${ }^{1}$, Riccardo Artoni ${ }^{2 *}, F u$-Ling Yang ${ }^{1 \dagger}$, and Patrick Richard ${ }^{2}$ \\ ${ }^{1}$ Department of Mechanical Engineering, National Taiwan University, Taipei 10617, Taiwan \\ ${ }^{2}$ MAST-GPEM, Univ Gustave Eiffel, IFSTTAR, F-44344 Bouguenais, France
}

\begin{abstract}
This work employs a Landau-Ginzburg-type nonlocal rheology model to account for shear localization in a wall-bounded dense granular flow. The configuration is a 3D shear cell in which the bottom bumpy wall moves at a constant speed, while a load pressure is applied at the top bumpy wall, with flat but frictional lateral walls. At a fixed pressure, shear zones transit from the top to the bottom when increasing lateral wall friction coefficient. With a quasi-2D model simplification, asymptotic solutions for fluidization order parameters near the top and bottom boundaries are sought separately. Both solutions are the Airy function in terms of a depth coordinate scaled by a characteristic length which measures the width of the corresponding shear zone. The theoretical predictions for the shear zone widths against lateral wall friction coefficient and load pressure agree well with data extracted from particle-based simulation for the flow.
\end{abstract}

\section{Introduction}

Granular flow is ubiquitous in many industrial applications (grain transport and storage) and geophysical events (landslides and debris flows), yet a unified description remains a challenge. Unlike usual fluids, granular material yields and flows when the ratio of bulk shear to normal stress exceeds a certain critical value [1]. Consequently, the material deforms nonuniformly by means of shear bands near boundaries $[1-5,12,13]$. Such a shear localization phenomenon is often described by a constant yield bulk friction for the flow to occur $[1,3]$. However, many studies have shown that solid boundary can have a strong influence on shear localization $[2,4,5,12,13]$. It is interpreted as nonlocal effects that wall disturbances can fluidize regions below yield through long-range transport along grain-scale contact network [5-11]. Several nonlocal models with diffusion order parameter have been proposed to capture wall shear localization in 2D confined flows [6-11].

Recently, Artoni and Richard show that shear localization can occur at several locations along the depth in a 3D rectangular parallelepiped periodic cell flow [12] or in a 3D torsional shear cell flow [13]. They found that the location of shear localization strongly depends on lateral wall friction coefficient. While a simplified stress analysis can provide a basic understanding of the effect of lateral wall friction on shear localization patterns [14], a full description for the flow rheology is desired.

In this paper, a Landau-Ginzburg-type nonlocal rheology model is used to describe shear localization transition in the 3D shear cell flow studied in [12]. The asymptotic method and the width-averaging technique are employed to reduce the model so that analytical solutions are possible to predict the effect of lateral wall friction and other parameters on shear localization and its transition.

\section{Shear localization transition}

The configuration of the wall-bounded granular flow in [12] is shown in Fig.1(a), which is a rectangular channel with a periodic boundary condition in the main flow direction in $x$, with two bumpy walls at the top and at the bottom, and two lateral flat but frictional walls (in the $y$ direction). Gravity acts on the system along the $z$ direction. The flow is driven by applying a wall speed $V$ at the bottom along the $x$-direction. The top wall is only free to move in the $z$-direction according to the pressure applied on it. By dimensional analysis, the flow is controlled by three parameters: (1) particle Froude number, $\mathrm{Fr} \equiv V /(g d)^{1 / 2}(2)$ loading parameter $M \equiv P_{0} / \rho g H$, which compares the loading pressure on the top wall $P_{0}$ and a pressure due to total particle weight (3) particlelateral wall friction coefficient $\mu_{p w}$. Here, $\rho$ denotes the bulk density, $g$ the acceleration of gravity, $d$ the particle diameter, and $H$ the material height. In [12], profiles along $z$ are computed by performing slice-averaging with a thickness $2 d$ in the $z$-direction. The flow behavior is found insensitive to the Froude number but strongly depend on the load parameter and the lateral wall friction, which can be categorized into three regimes

\footnotetext{
* Corresponding author: riccardo.artoni@univ-eiffel.fr

$\uparrow$ Corresponding author: fulingyang@ntu.edu.tw
}

A video is available at https://doi.org/10.48448/aae5-9m67 
according to the depth velocity profiles shown in Fig.1(b):

A. High $\boldsymbol{M}$ and high $\boldsymbol{\mu}_{p w}$. Shear localized at the bottom. B. Low $M$ and low $\mu_{p w}$. Shear localized at the top. C. Low $M$ and intermediate $\mu_{p w}$. A central plug flow zone with two shear zones at the walls.

Figure 1(c) shows the profiles of effective bulk friction coefficient $\mu \equiv \sigma_{z x}{ }^{b} / P$, corresponding to the depth velocity profiles in Fig.1(b), where $\sigma_{z x}{ }^{b}$ is a bulk deviatoric shear stress on the $z$-face acting in the $x$-direction, and $P \equiv\left(\sigma_{x x}^{b}+\sigma_{y y}{ }^{b}+\sigma_{z z}{ }^{b}\right) / 3$ is a bulk isotropic pressure. It displays that increasing $\mu_{p w}$ weakens $\mu$ near the top but enhances $\mu$ near the bottom. Such a transition of the bulk friction gradient is speculated to cause the flow regime transition and has been qualitatively captured by a simplified stress analysis in [14] based on force balances between gravity and lateral wall friction. In [13], an inertial $\mu(I)$-rheology model $[1,3]$ is numerically solved to predict the detailed flow profiles in the shear cell while the predictions show rate-dependent depth velocity profiles, inconsistent with the rate-independent profiles observed in the simulations. Indeed, the rateindependence characterizes quasistatic granular rheology and suggests that the finite shear zones in the three regimes are generated by nonlocal processes driven by wall shearing along grain contact network. While several nonlocal approaches have been put forward to account for similar long-range transport phenomenon [6-11,15], here we adopt a simple yet effective Landau-Ginzburg approach which introduces a diffusion-reaction equation describing shear-induced fluidization processes, which has been shown to well capture the nonlocal rheology of $2 \mathrm{D}$ free-surface inclined flows and confined sheared flows $[6,10,11]$.
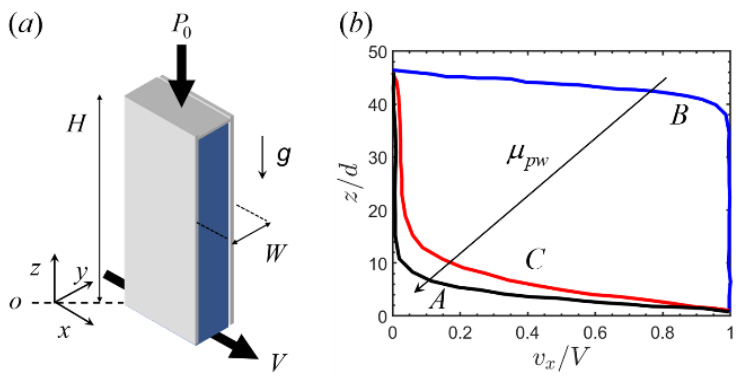

(c)

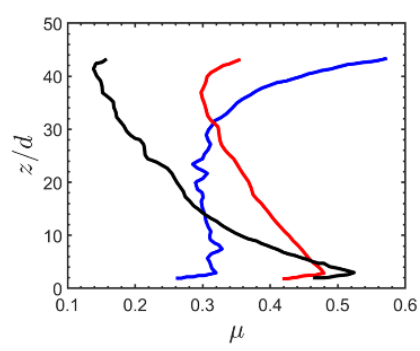

Fig. 1. (a) Wall-bounded flow configuration. Profiles of (b) normalized depth velocity and (c) effective bulk friction coefficient with varying lateral wall friction for $\mathrm{Fr}=10$ and $M=0.2$ (data are extracted from [12]).

\section{Model formulation}

\subsection{Landau-Ginzburg order-parameter model}

A phenomenological Landau-Ginzburg order parameter model is introduced and simplified for the current flow condition as follows. The basic idea of the model is to treat the transition of granular non-sheared and sheared states as a solid-fluid phase transition. In this sense, thermal temperature in the original Landau-Ginzburg framework is replaced with the effective friction coefficient $\mu$ which controls the occurrence of granular "phase" change due to material yield. Hence, a LandauGinzburg differential equation describing how a shearinduced fluidization state evolves in space and time under a given $\mu$-field can be written as

$$
t_{0} \frac{D \lambda}{D t}=l^{2} \nabla^{2} \lambda-\frac{\partial}{\partial \lambda} F_{l o c}(\lambda, \mu) .
$$

Here, $\lambda$ denotes an order parameter (OP) measuring the degree of shear-induced fluidization at a location $\boldsymbol{r}$ and time $t, t_{0}$ represents a relaxation timescale, $l$ a microscopic lengthscale $(\sim d)$. The source term corresponds to the derivative of a coarse-grain Landau free energy density, which can be expanded to be

$$
-\partial_{\lambda} F_{l o c}(\lambda, \mu)=\left(\mu-\mu_{c}\right) \lambda+A \lambda^{1+\alpha}-B \lambda^{1+\beta}+\text { h.o.t., }
$$

where $\mu_{c}$ denotes a critical friction coefficient at which the material yields, and $\alpha$ and $\beta$ are positive exponents with $\beta>\alpha>0$. Note that different forms of $F_{l o c}$ have been proposed in literature $[6,10,11]$. For a steady, quasistatic flow considered here, the unsteady term and the higher-order source terms in Eq.(2) are dropped to get

$$
0 \approx l^{2} \nabla^{2} \lambda+\left(\mu-\mu_{c}\right) \lambda
$$

This simplified equation describes how a steady flow zone above yielding $\mu>\mu_{c}$ fluidize neighboring zones below yielding $\left(\mu<\mu_{c}\right)$. The nonlocal Laplacian term in Eq.(3) hence extends the classical Mohr-Coulomb plasticity theory which indicates zero thickness for shear zone. Note that in order to predict flow profiles the OP has been linked to a variety of rheological parameters such as the fraction of fluid stress contribution [6], a granular fluidity, the inverse of viscosity scaled by pressure $[8,10]$, and the inertial number $I$, a scaled shear rate by pressure indicating the quasistatic and the inertial regimes [9,11]. Also note that the form of Eq.(3) is identical to the steady-state-only granular fluidity theory in [8] without the inertial-rheology contribution. Yet the effectiveness of these OP definitions in the current geometry needs to be further investigated via systematically comparing the predicted profiles to the flow and the stress data from the simulations. As a preliminary study here, we aim to employ the OP model (3) to derive the scaling lengths of the shear zones and examine the predictions using the width data of the shear zones extracted by exponential fit to the depth velocity profiles in simulation.

\subsection{Width-averaged stress model}

To solve the nonlocal model (3), the effective friction profile is required. To simplify the problem, the widthaveraged technique is employed to reduce the momentum balance equations. This treatment is reasonable as long as the flow height, $H$, is far greater than the channel width, $W$. In our simulations, the ratio 
$K \equiv H / W=4.5$ which is acceptable. The width-averaged momentum equations in $x$ and $y$ are given by

$$
\frac{2 \sigma_{y x}{ }^{w}}{W}+\frac{d}{d z}<\sigma_{z x}>=0, \frac{d}{d z}<\sigma_{z z}>=-\rho g,
$$

where $\sigma_{y x}{ }^{\mathrm{w}}$ is a deviatoric shear stress on the lateral walls acting in the $x$-direction, $\sigma_{z x}$ deviatoric shear stress on the $z$-face acting in the $x$-direction, and $\sigma_{z z}$ normal stress in the $z$-direction, and $\langle\ldots\rangle$ denotes the width-averaging in the $y$-direction. To avoid confusion with the lateral walls, $z=H$ and $z=0$ are referred to as the top and bottom boundaries hereinafter. The averaged normal stress $\left\langle\sigma_{z z}\right\rangle$ is assumed isotropic and is prescribed to be $\left\langle\sigma_{z z}\right\rangle(H)=P_{0}$ at the top boundary. The effective friction and the lateral wall friction coefficient are defined as

$$
\mu \equiv \frac{<\sigma_{z x}>}{<\sigma_{z z}>}, \quad \mu_{w} \equiv \frac{\sigma_{y x}{ }^{w}}{<\sigma_{z z}>},
$$

respectively. For the sake of simplicity, here we assume the lateral wall friction is equal to the particle-wall friction, i.e., $\mu_{w}=\mu_{p w}$. Note however that this assumption may be inappropriate for large $\mu_{p w}$ where significant wall friction weakening, i.e., $\mu_{w}<\mu_{p w}$, is observed everywhere in the system as shown in [12]. With this assumption, Eq. (4a,b) and (5a,b) can be solved to be

$$
\mu=\frac{C-2 \mu_{p w} K(1+M)(z / H)+\mu_{p w} K(z / H)^{2}}{1+M-z / H},
$$

where $C=M \mu_{T}+K(1+2 M) \mu_{p w}=(1+2 M) \mu_{B}$ with $\mu_{T}=\mu(H)$ and $\mu_{B}=\mu(0)$ denoting the boundary $\mu$ at the top and bottom, respectively.

\section{Near-boundary asymptotic analysis}

The asymptotic solutions near the top and bottom boundaries are sought. The boundary conditions for the OP are prescribed to be no-flux at the two solid boundaries, meaning that there is no flow of shearinduced fluidization across the boundaries. In addition, the OP is assumed to vanish as approaching the central zone. Similar OP boundary conditions have been adopted in 2D nonlocal modelling for other confined flows [16]. Assuming the widths of the shear zones (scaled with $l$ ) are asymptotically smaller than the flow height, Eq.(3) and (6) and the above boundary conditions can be reduced to two separate asymptotic problems as follows:

- Near the top boundary with $z^{*} \equiv(H-z) / l \sim 1$ :

$$
\begin{gathered}
0=\frac{d^{2} \lambda^{(T)}}{d z^{* 2}}+\left(\mu-\mu_{c}\right) \lambda^{(T)} \\
\mu=\mu_{T}-\Delta \mu_{T} z^{*}+\text { h.o.t } \\
\frac{d \lambda^{(T)}}{d z^{*}}=0 \text { at } z^{*}=0 \text { and } \lambda^{(T)} \rightarrow 0 \text { as } z^{*} \rightarrow \infty . \quad(7 \mathrm{c},
\end{gathered}
$$

- $\quad$ Near the bottom boundary with $\hat{z} \equiv z / l \sim 1$ :

$$
\begin{gathered}
0=\frac{d^{2} \lambda^{(B)}}{d \hat{z}^{2}}+\left(\mu-\mu_{c}\right) \lambda^{(B)}, \\
\mu=\mu_{B}-\Delta \mu_{B} \hat{z}+\text { h.o.t, } \\
\frac{d \lambda^{(B)}}{d \hat{z}}=0 \text { at } \hat{z}=0 \text { and } \lambda^{(B)} \rightarrow 0 \text { at } \hat{z} \rightarrow \infty .
\end{gathered}
$$

Here, $\Delta \mu_{T}$ and $\Delta \mu_{B}$ measure the significances of the spatial nonuniformity of $\mu$ in the shear zones at the top and bottom, respectively:

$$
\begin{aligned}
& \left.\Delta \mu_{T} \equiv \frac{d \mu}{d z}\right|_{z=H} l=\frac{\mu_{T}}{M h}-\frac{2 \mu_{p w} K}{h}, \\
& \left.\Delta \mu_{B} \equiv \frac{d \mu}{d z}\right|_{z=0} l=\frac{2 \mu_{p w} K}{h}-\frac{\mu_{B}}{(1+M) h},
\end{aligned}
$$

where $h \equiv H / l$ is a scaled flow height. Zero $\Delta \mu_{T}$ and $\Delta \mu_{B}$ occur with uniform $\mu$-profile for infinite loading (infinite $M$ ) and perfectly smooth lateral wall (zero $\mu_{p w}$ ).

It can be shown that Eq. $(7 a, b)$ and $(8 a, b)$ reduce to the Airy equation after appropriate transformation of the variables, and their solutions subject to the conditions $(7 \mathrm{c}, \mathrm{d})$ and $(8 \mathrm{c}, \mathrm{d})$ are the Airy function of the first kind:

$$
\begin{aligned}
& \lambda^{(T)}\left(z^{*}\right)=\lambda_{T} A i\left(\frac{\mu_{c}-\mu_{T}}{\Delta \mu_{T}^{2 / 3}}+\frac{z^{*}}{\Delta \mu_{T}^{-1 / 3}}\right), \\
& \lambda^{(B)}(\hat{z})=\lambda_{B} A i\left(\frac{\mu_{c}-\mu_{B}}{\Delta \mu_{B}^{2 / 3}}+\frac{\hat{z}}{\Delta \mu_{B}^{-1 / 3}}\right),
\end{aligned}
$$

with

$$
\mu_{T}=\mu_{c}-\Delta \mu_{T}^{2 / 3} q_{c}, \quad \mu_{B}=\mu_{c}-\Delta \mu_{B}^{2 / 3} q_{c} .
$$

Here, $q_{c}=-1.019$ is a numeric value at the first local maximum of $A i(q)$ along the negative $q$ axis [ $\left.A i{ }^{\prime}\left(q_{c}\right)=0\right]$, and $\lambda_{T}$ and $\lambda_{B}$ denote the OP at the boundaries. Significantly, Eq.(11) and (12) indicate two lengths associated with the widths of the boundary shear zones:

$$
\delta_{T}=\Delta \mu_{T}{ }^{-1 / 3} l, \quad \delta_{B}=\Delta \mu_{B}{ }^{-1 / 3} l .
$$

Manipulating Eq.(9), (10), and (13-16), we derive the following implicit solutions for the shear zone widths:

$$
\begin{gathered}
\frac{\delta_{T}}{l}=\left[\left(1+\frac{q_{c} \delta_{T}}{M h l}\right)\left(\frac{\mu_{c}-2 K M \mu_{p w}}{h M}\right)\right]^{-1 / 3}, \\
\frac{\delta_{B}}{l}=\left[\left(1-\frac{q_{c} \delta_{B}}{(1+M) h l}\right)\left(\frac{2 K(1+M) \mu_{p w}-\mu_{c}}{h(1+M)}\right)\right]^{-1 / 3} .
\end{gathered}
$$

In order to check these expressions, we performed molecular dynamics simulations of the confined shear flow with the LIGGGHTS software [17], for 10000 spheres (corresponding roughly to $H=45 d$ ), one value of the Froude number $(\mathrm{Fr}=1)$, and different values of the dimensionless load $(M=0.1,1,10)$ and the particle-wall friction coefficient $\left(\mu_{p w}=0-0.4\right)$. A linear spring-dashpot contact model with friction was used, with normal stiffness $k_{n}=8 \times 10^{5} \mathrm{mg} / \mathrm{d}$, tangential stiffness $k_{t}=2 / 7 k_{n}$, restitution coefficients $e_{n}=0.7, e_{t}=0$, and interparticle friction $\mu_{p}=0.5$. From these simulations, we estimated the top and bottom shear zone widths as the exponential decay lengths obtained by exponential fit to the velocity data.

Figure. 2 compares model predictions by Eq.(17) and (18) (lines) to the simulation data (symbols) for the top and bottom shear zone widths against the lateral wall friction coefficient $\mu_{p w}$ with the load parameter $M=0.1,1$, and 10 . The model predictions agree well with the data. The results show that, for a given $M$, increasing $\mu_{p w}$ results in an increase in $\delta_{T}$ but a decrease in $\delta_{B}$. As $M$ is increased, $\delta_{T}$ drastically diverges at low $\mu_{p w}$ while $\delta_{B}$ becomes more 
uniform along the $\mu_{p w}$-axis. Since the growth of shear zone manifests a nonlocal behavior that particles are strongly correlated over a long distance like solids, the above two features indicate the shear zone transition from the flow regime A (low $\mu_{p w}$ and $M$ ) to B (high $\mu_{p w}$ and $M$ ) introduced in Sec.2. On the other hand, for low $M=0.1$ and 1 , there exists regions with intermediate $\mu_{p w}$ where the $\delta_{T}$ and $\delta_{B}$ solution branches overlap. Such overlap regions characterize the flow regime $C$ where the two shear zones coexist. This may also account for the growth of the deviations between the model and the data in the overlap regions as $M$ decreases since the boundary asymptotic expansions no longer hold when shear develops across the entire depth.

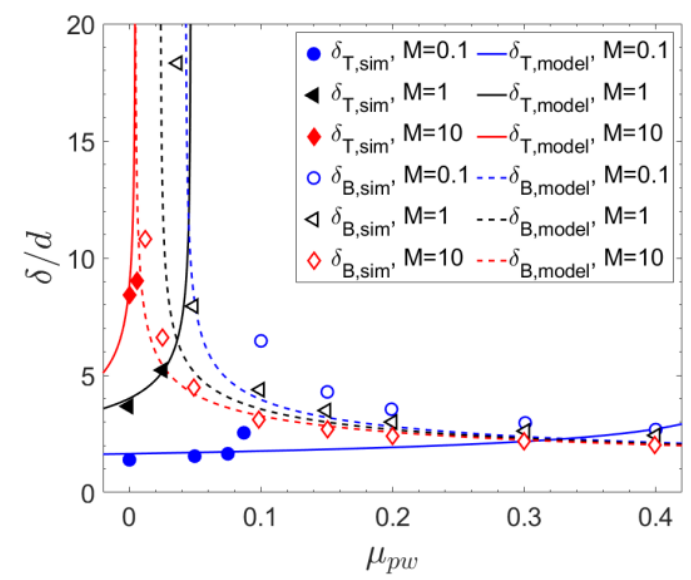

Fig. 2. Shear zone width versus lateral wall friction coefficient with various load parameter $M=0.1,1,10$. The subscripts T and B denote the top wall and the bottom wall, respectively. Simulation data $\delta_{T, \text { sim }}$ and $\delta_{B, \text { sim }}$, shown in symbols, are exponential decay lengths extracted by exponential fit to depth velocity data. Model prediction $\delta_{T, \text { model }}$ and $\delta_{B, \text { model }}$ are shown in lines given by Eq.(17) and (18), where a typical value $\mu_{c}=0.38$ is chosen and $l / d=0.79$ is an averaged fitted value.

\section{Conclusion}

We have analytically solved a Landau-Ginzburg nonlocal rheology model to account for the shear localization in a 3D wall-bounded sheared granular flow shown in Fig.1. With the steady quasistatic flow condition and the width-averaged effective friction profiles, the boundary asymptotic solutions for the fluidization order parameters are solved to be the Airy function of the first kind. The solutions reveal two decay lengths associated with the shear zone widths at the top and bottom. We have shown that the width solutions agree well with the data extracted from the particlebased simulations and can account for the observed flow regime transition.

A key conclusion draw from the present study is that the formation of the shear zones strongly depends on the extent of nonuniformity in the effective friction coefficient $\mu$ near the solid boundaries, as shown in Eq. (15) and (16). According to the nonlocal model (3), if $\mu$ is significantly nonuniform near a boundary (large $\Delta \mu_{T}$ or $\left.\Delta \mu_{B}\right)$, it allows the coexistence of a near-boundary flow region above yielding $\left(\mu>\mu_{c}\right)$ and a far-field creep region below yielding $\left(\mu<\mu_{c}\right)$. On the contrary, when $\mu$ becomes uniform near the boundary, $\mu$ tends the yielding point $\mu_{c}$ in the two regions so that the material near the boundary becomes solidlike and hence the shear zone vanishes. For the wall-bounded flow considered here, increasing the lateral wall friction and the loading weight result in a locally uniform $\mu$ profile near the top (smaller $\Delta \mu_{T}$ ) but enhances the gradient of $\mu$ near the bottom (higher $\Delta \mu_{B}$ ), causing the shear zone to transit from the top to the bottom [see Fig.1(b)]. The current nonlocal model is consistent with the physical arguments based on the stress analysis in [14] and further captures the rheological features beyond the scope of the inertial $\mu(I)$ rheology [13].

The authors would like to acknowledge the financial support from Ministry of Science and Technology of Taiwan and Campus France, under the Bilateral Orchid Project MOST 107-2911-I-002-536 / Campus France 40943RF, and MOST 106-2628-E-002-012-MY3.

\section{References}

[1] GDR-MIDI, Eur. Phys. J. E 14, 341-365 (2004)

[2] N. Taberlet, P. Richard, A. Valance, W. Losert, J. M. Pasini, J. T. Jenkins, R. Delannay, Phys. Rev. Lett. 91, 264301 (2003)

[3] P. Jop, Y. Forterre, O. Pouliquen, J. Fluid Mech. 541, 167-192 (2005)

[4] P. Richard, A. Valance, J.-F. Metayer, P. Sanchez, J. Crassous, M. Louge, R. Delannay, Phys. Rev. Lett. 101, 248002 (2008)

[5] K. A. Reddy, Y. Forterre, O. Pouliquen, Phys. Rev. Lett. 106, 108301 (2011)

[6] I. S. Aranson, L. S. Tsimring, Phys. Rev. E 65, 061303 (2002)

[7] O. Pouliquen, Y. Forterre, Phil. Trans. Royal Soc. A 367, 5091 (2009)

[8] K. Kamrin and G. Koval, Phys. Rev. Lett. 108, 178301 (2012)

[9] M. Bouzid, M. Trulsson, P. Claudin, E. Clément, B. Andreotti, Phys. Rev. Lett. 111, 238301 (2013)

[10] K. Kamrin, D. L. Henann, Soft Matt. 11, 179-185 (2015)

[11] K.-L. Lee, F.-L. Yang, Phys. Rev. E 96, 062909 (2017)

[12] R. Artoni, P. Richard, Phys. Rev. Lett. 115, 158001 (2015)

[13] R. Artoni, P. Richard, Comput. Part. Mech. 5, 3-12 (2018)

[14] R. Artoni, A. Soligo, J. Paul and P. Richard, J. Fluid Mech. 849, 395-418 (2018)

[15] J. T. Jenkins, D. Berzi, Granular Matter 12, 151-158 (2010)

[16] D. Liu, D. L. Henann, Soft Matter 14, 5294 (2018)

[17] K. Christoph, G. Christoph, H. Alice, A. Stefan, P. Stefan, Models, algorithms and validation for opensource DEM and CFD-DEM, - Progress in Computational Fluid Dynamics, An Int. J. - Vol. 12, No.2/3, 140-152 (2012) 